\title{
Processing of Electroactive Ceramics in the Transmission Electron Microscope
}

\author{
Jenna Wardini ${ }^{1}$, Jairo Gonzalez ${ }^{1}$, George Harrington ${ }^{2}$ and William Bowman ${ }^{3}$
}

${ }^{1}$ Department of Materials Science and Engineering, University of California, Irvine, CA, USA, United States, ${ }^{2}$ Next-Generation Fuel Cell Research Centre, Kyushu University, Fukuoka, Japan, United States, ${ }^{3}$ Department of Materials Science and Engineering, University of California, Irvine, CA, USA, Irvine, California, United States

Mixed ion and electron conducting (MIEC) ceramics are widely studied for use in a variety of electrochemical cells. Lanthanum Strontium Manganite (LSM) is a prototypical MIEC, often used as a cathode material in commercially available solid oxide cells (SOC). SOC efficiency largely depends on the ease of oxygen transport in the MIEC, as it plays a critical role in the oxygen exchange process $\left(\mathrm{O}_{2} \leftrightarrow \mathrm{O}^{2-}\right)$. Previous work shows that grain boundaries (GBs) in polycrystalline LSM can facilitate an oxygen diffusivity up to five orders of magnitude greater than that of the crystalline grain interior [1]. As a result, GBs and other crystalline defects offer pathways for fast oxygen transport, depending on the local cationic and anionic composition which affect the local point defect concentration [2]. By tuning the nature of the GB network in LSM, the diffusivity of oxygen and therefore the ionic conductivity of these films can be controlled by tuning the microstructure.

The present work aims establish a relationship between characteristics of the GB network (e.g., grain size, GB atomic structure and local defect chemistry) and GB-facilitated $\mathrm{O}^{2-}$ transport. Here, amorphous $\mathrm{La}_{0.8} \mathrm{Sr}_{0.2} \mathrm{MnO}_{3}$ (LSM82) films deposited by pulsed laser deposition (PLD) were heat treated and crystallized in situ in the transmission electron microscope. Scanning and transmission electron microscopy ((S)TEM) paired with electron energy loss and energy dispersive spectroscopies (EELS, EDS) were used to simultaneously monitor the structural (e.g., grain size, crystal structure) and chemical (e.g., elemental distribution, and cation bonding environment) state of the film.

In this work we investigate the effects of annealing thin $(30 \mathrm{~nm})$ LSM82 films at different oxygen partial pressures $\left(\mathrm{pO}_{2}\right)$, microscope vacuum and air, and explore how characteristics of the as-deposited amorphous films affect the resulting microstructure (Fig. 2). In both vacuum and air, the amorphous LSM82 film does not form the single phase perovskite structure predicted by thermodynamic calculations for bulk systems at the given conditions $\left(900{ }^{\circ} \mathrm{C}, \log \left(\mathrm{pO}_{2} / \mathrm{atm}\right)_{\text {vacuum }}=-10.4, \log \left(\mathrm{pO}_{2} / \mathrm{atm}\right)_{\text {air }}=-0.7\right)$ [3]. Rather, upon vacuum annealing we observe multi-cation segregation from the crystallized phase $\left(\sim 700{ }^{\circ} \mathrm{C}\right)$ followed by the formation of their respective oxides $\left(\geq 800{ }^{\circ} \mathrm{C}\right)$ accompanied by multiple other decomposition products. We attribute the accelerated decomposition of the initially crystallized phase both to the low- $\mathrm{pO}_{2}$ annealing environment, as well as enhanced oxygen loss and depressed melting points due to the reduced dimensionality of the thin film compared to the bulk [4]. Further, the fact that the air anneal also results in a multi-phase system (albeit less complex) implies that thin film effects are substantial and must be considered to form the target phase.

Temperature-dependent electrical measurements reveal a general increase in conductivity with temperature which indicates oxygen transport likely occurs. However, since the phases resulting from both the vacuum and air anneal are not a single phase perovskite, the electrical behavior cannot be correlated to characteristics of the GB network as intended. Future work will aim to find in situ processing conditions that form a single phase LSM82 perovskite. These unexpected phase transformations pose a considerable challenge for in situ thin film processing of multicomponent systems, not only because the anticipated structure may not be achieved, but also because phase identification is critical to understanding the film evolution. This is particularly challenging in nanocrystalline multiphase thin film systems due to the difficulty of isolating a single-phase for analysis. Here, we attempt to address this challenge with a variety of approaches to generate a model of the phase decomposition of LSM82 thin films in various annealing environments. 

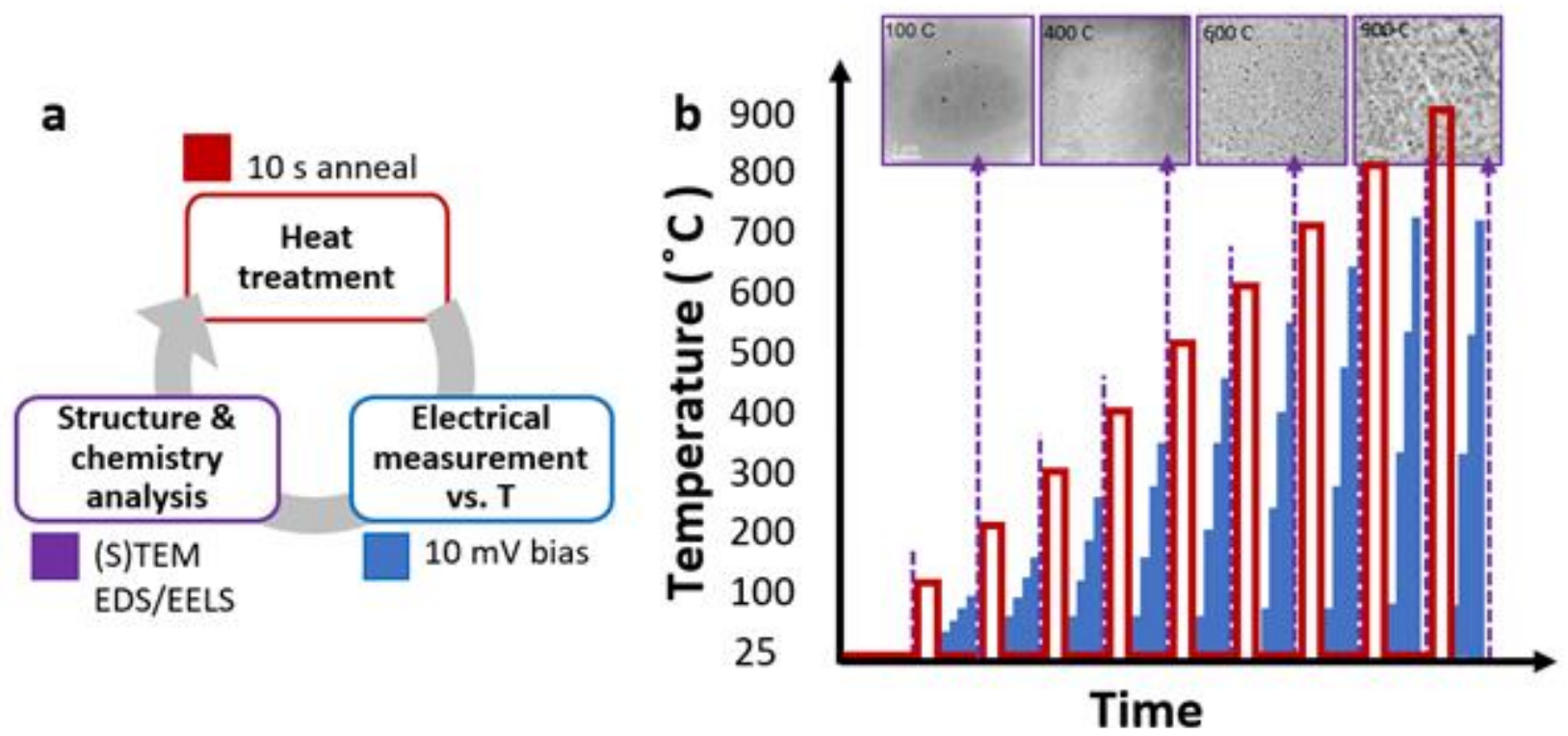

Figure 1. Figure 1: a) In situ processing workflow and b) Incremental pulse annealing (red curve) followed by temperature-dependent electrical measurements (blue) to extract activation energies for charge carrier transport with examples of film microstructure taken after each annealing step.
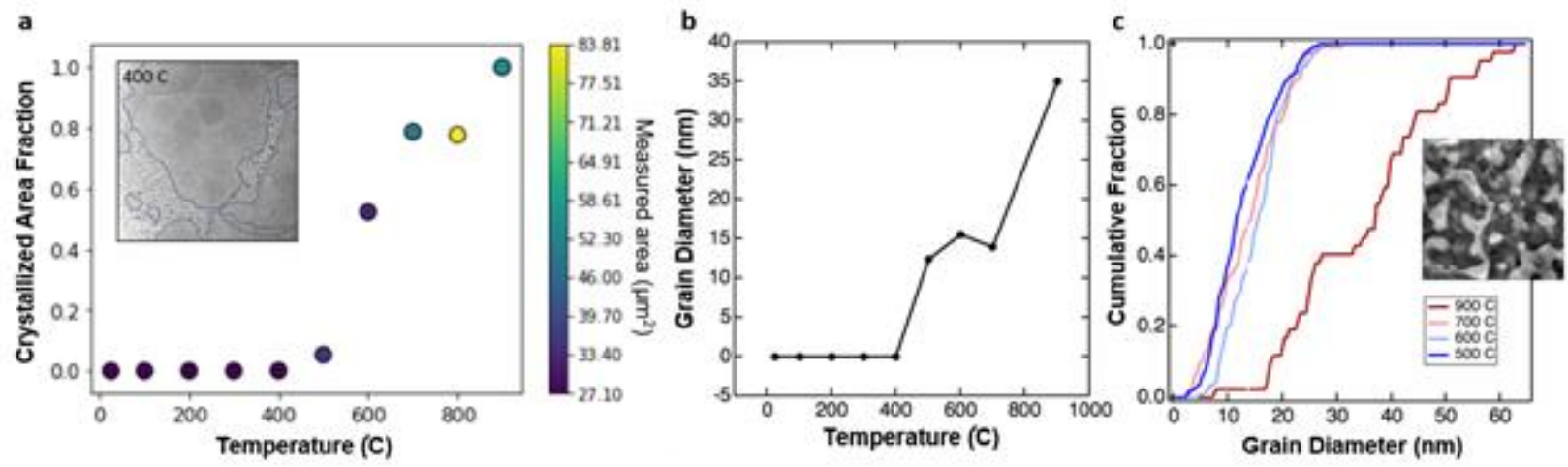

Figure 2. Figure 2: a) Crystallized area fraction as a function of temperature showing delayed crystallization onset in regions of the as-desposited film with greater mass-thickness. Inset TEM image taken after annealing to $400 \mathrm{C}$, blue lines indicate the barrier between thick and thin regions. b) Grain growth with temperature and c) Multimodal grain size distributions arising at higher temperature to decomposition, shown in the HAADFSTEM image where at least two compositionally distinct regions are shown.

References

[1] De Souza, R.A. et al. Materials Letters 43, 43-52 (2000)

[2] Chiabrera, F. et al. Advanced Materials 31 (2019)

[3] Cherepanov, V.A. Journal of Solid State Chemistry 134, 38-44 (1997)

[4] Gromov, Dmitry G. et al. Thermodynamics - Physical Chemistry of Aqueous Systems, Juan Carlos MorenoPiraján, IntechOpen, (2011) 